\title{
Bioaccesibilidad de compuestos antioxidantes de diferentes variedades de frijol (Phaseolus vulgaris L.) en México, mediante un sistema gastrointestinal in vitro
}

Bioaccessibility of antioxidant compounds from different bean varieties (Phaseolus vulgaris L.) in Mexico, through an in vitro gastrointestinal system

Liliana Maribel Pérez-Perez', Carmen Lizette Del Toro Sánchez', Esteban Sánchez Chavez², Ricardo Iván González Vega', Aline Reyes Díaz', Jesús Borboa Flores', Juan Manuel Soto Parra ${ }^{3}$, María Antonia Flores-Cordova ${ }^{3 *}$

Universidad de Sonora, Rosales y Niños Héroes S/N, 83000, Hermosillo, Son, México.

2 Centro de Investigación en Alimentación y Desarrollo, A.C. Unidad Delicias. Av 4a. Sur 3825. Delicias, Chihuahua. México.

Universidad Autónoma de Chihuahua, Escorza 900. Chihuahua, Chih. México.

\section{RESUMEN}

El frijol tiene alto contenido fenólico y actividad antioxidante, por ello es importante conocer cuánto se libera de estos compuestos de la matriz alimentaria y es aprovechado en el organismo. Por lo tanto, el objetivo de este trabajo fue determinar la bioaccesibilidad de compuestos antioxidantes de diferentes variedades de frijol (Phaseolus vulgaris L.) mediante un sistema gastrointestinal in vitro. Se realizó la cuantificación de compuestos fenólicos, flavonoides y capacidad antioxidante (DPPH, ABTS y FRAP) de 6 cultivares: Negro, Flor de Mayo, Patol, Pinto Saltillo, Teapa y Peruano, antes y después del cocimiento. Adicionalmente, se evaluó la digestión gastrointetinal in vitro en frijol cocido. Los resultados obtenidos de las variedades de frijol Teapa y Peruano, presentaron mayor rendimiento de extracto crudo, y un incremento del contenido fenólico. El frijol cocido, mostró un incremento en el contenido de flavonoides en las variedades Teapa, Peruano y Pinto Saltillo, así como mayor capacidad antioxidante, en las variedades Negro, Flor de mayo, Teapa y Peruano. La concentración inhibitoria media $\left(\mathrm{IC}_{50}\right)$ la alcanzaron Teapa, Pinto Saltillo y Flor de Mayo $(106.45,154.72$ y $127.16 \mu \mathrm{g} / \mathrm{mL}$ respectivamente). La digestión gastrointestinal in vitro dio como resultado mayor bioaccesibilidad para compuestos fenólicos, resaltando las variedades Teapa, Pinto Saltillo y Flor de mayo. Palabras clave: Compuestos fenólicos, antioxidantes, bioaccesibilidad, digestibilidad.

\section{ABSTRACT}

Beans have high phenolic content and antioxidant activity, so it is important to know how much of these compounds is released from the food matrix and is used in the body. Therefore, the objective of this work was to determine the antioxidant compounds bioavailability on different bean varieties (Phaseolus vulgaris L.) through an in vitro gastrointestinal system. Quantification of phenolic compounds, flavonoids and antioxidant capacity (DPPH, ABTS and FRAP) of 6 cultivars (Black, Flor de Mayo, Patol, Pinto Saltillo, Teapa and Peruvian) was carried out, before and after cooking. Additionally, in vitro gastrointetinal digestion in cooked beans was evaluated. The results obtained from the Teapa and Peruvian bean varieties showed a higher yield of crude extract and an

*Autor para correspondencia: María Antonia Flores Cordova

Correo electrónico: mariflor_556@hotmail.com

Recibido: 29 de abril de 2019

Aceptado: 3 de julio de 2019 increase in phenolic content. Cooked beans showed an increase in flavonoid content in the Teapa, Peruvian and Pinto Saltillo varieties, as well as greater antioxidant capacity, in the Negro, Flor de Mayo, Teapa and Peruvian varieties. The mean inhibitory concentration $\left(\mathrm{IC}_{50}\right)$ was reached by Teapa, Pinto Saltillo and Flor de Mayo (106.45, 154.72 and $127.16 \mu \mathrm{g} / \mathrm{mL}$ respectively). The gastrointestinal digestion in vitro resulted in greater bioavailability for phenolic compounds, highlighting the Teapa, Pinto Saltillo and Flor de Mayo varieties.

Keywords. Phenolic compounds, antioxidants, bioaccessibility, digestibility.

\section{INTRODUCCIÓN}

El frijol (Phaseolus vulgaris L.) es conocido como una de las principales fuentes de nutrientes en todo el mundo, ocupando un lugar importante en la dieta humana, debido al contenido de proteínas, carbohidratos, minerales y vitaminas (Diaz et al., 2010; Celmeli et al., 2018). Además, tiene compuestos fenólicos como ácido ferúlico, p-cumárico y gálico, así como gran variedad de flavonoides como son antocianinas, flavonoles y proantocianidinas, los cuales le confieren actividad biológica antioxidante. Los antioxidantes se relacionan con la disminución de enfermedades crónicas, ya que pueden contrarrestar las especies reactivas de oxígeno, inhibiendo los mecanismos oxidativos que conducen a la degeneración celular, confiriendo con esto grandes efectos benéficos a la salud (Mojica et al., 2014). Lo anterior, depende de la cantidad consumida de los compuestos fenólicos, de su bioaccesibilidad (fracción máxima liberada de la matriz del alimento) y su biodisponibilidad (fracción que alcanza la circulación para ejercer acción en el organismo) (Manach et al., 2004; Gutiérrez-Grijalva et al., 2016), el cual implica un proceso complejo de varias etapas diferentes: fases de liberación, absorción, distribución, metabolismo y eliminación (Maarit et al., 2013).

Los compuestos fenólicos, son compuestos naturales, de grupos fenilo hidroxilados, que se encuentran en formas glucosiladas, esterificados o polimerizados. Cuando son ingeridos, son reconocidos por el cuerpo humano como xenobióticos. Los polifenoles de bajo peso molecular tales como estructuras monoméricas y diméricas, pueden ser 
absorbidos por el intestino delgado (Karas et al., 2016). Se han llevado a cabo estudios, en los cuales afirman que el $5-10 \%$ de la ingesta total se absorbe en el intestino delgado. El resto se acumula en el gran lumen intestinal, los cuales son excretados a través de la bilis y/o sometidos a las actividades enzimáticas de la comunidad microbiana intestinal (Cardona, 2013; Perez-Perez et al., 2018). Por lo tanto, los compuestos fenólicos podrían ser sensibles a condiciones de digestión y modificados en favorables estructuras promotoras de salud (Kahlon y Smith, 2007). Así mismo, los antioxidantes naturales pueden funcionar sinérgicamente para promover la salud humana, sin embargo, investigaciones sobre el efecto de la digestión gastrointestinal de los antioxidantes son escasos. Se han realizado algunos reportes de biodisponibilidad que señalan que los polifenoles pueden depender de su absorción en el intestino (Saura-Calixo et al., 2006). Así mismo, Laparra et al. (2008) evaluaron la bioaccesibilidad individual de polifenoles en frijol, exhibiendo valores altos de bioaccesibilidad. Soriano et al. (2015) reportaron una disminución de compuestos bioactivos y un aumento en la actividad antioxidante por efecto de la digestión in vitro en testa de frijol. Además, la bioaccesibilidad de los antioxidantes en la dieta es importante porque en la digestión gastrointestinal deben estar disponibles para su absorción, y así ayudar a proteger al intestino contra especies reactivas de oxígeno (Chia-Jung et al., 2013), así como a proteger la mucosa intestinal a través de actividades antioxidantes y antiinflamatorias (Gálvez et al, 2001).

Por todo lo anterior, el objetivo de este trabajo fue determinar la bioaccesibilidad de compuestos antioxidantes de diferentes variedades de frijol (Phaseolus vulgaris L.) producidos en México, mediante un sistema gastrointestinal in vitro.

\section{MATERIALES Y METÓDOS \\ Material vegetal}

Se utilizaron seis variedades de frijol en grano, representativos de Chihuahua (frijol Pinto Saltillo), Durango (frijol Flor de Mayo y frijol Patol), Morelos (Frijol Negro), Jalisco (Peruano) y Tabasco (frijol Teapa), en base a la preferencia de consumo (Figura 1). Las muestras se trasladaron al laboratorio en donde se procedió a realizar la extracción de los compuestos antioxidantes.

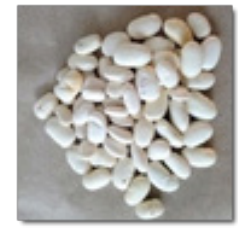

Patol

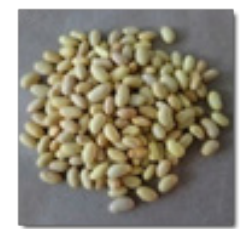

Peruano

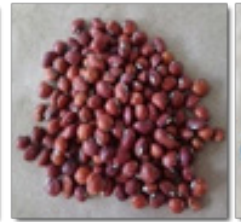

Teapa

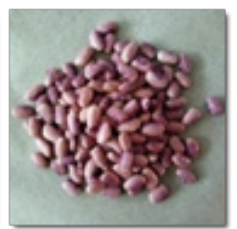

Flor de mayo

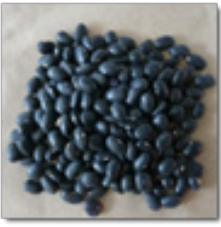

Negro

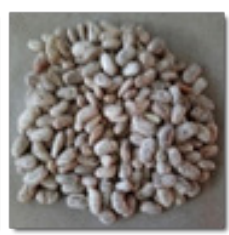

Pinto Saltillo
Figura 1. Semillas de diversos cultivares de Phaseolus vulgaris L.

\section{Extracción de los compuestos antioxidantes}

Los granos crudos de cada tipo de frijol se molieron y tamizaron (tamiz número 20) hasta obtener un tamaño de partícula de $0.84 \mathrm{~mm}$. Posteriormente, $15 \mathrm{~g}$ de muestra se mezclaron con $30 \mathrm{~mL}$ de metanol al $80 \%$, se agitó por una hora en un skhaker orbital KJ-201 BD para después ser centrifugados por $10 \mathrm{~min}$ a $4000 \times \mathrm{g}$ a $4{ }^{\circ} \mathrm{C}$. Se recuperaron los sobrenadantes y al residuo se volvió a aplicar por segunda ocasión el mismo procedimiento de extracción. Al final los dos sobrenadantes obtenidos se combinaron y se concentraron a presión reducida mediante rotavapor (Buchi, R-100 V) para la determinación del rendimiento de extracto obtenido. Posteriormente se resuspendieron en metanol hasta obtener una concentración de $0.178 \mathrm{~g}$ de extracto/mL. Las muestras se almacenaron a $-20^{\circ} \mathrm{C}$ para su posterior análisis.

Adicionalmente, se utilizaron muestras cocidas para el estudio. Para el cocimiento, los granos de frijol (80 g) se remojaron durante $10 \mathrm{~h}$ en agua destilada $(100 \mathrm{~mL})$, para posteriormente ser cocinados en una autoclave a $121{ }^{\circ} \mathrm{C}$ durante 30 min (modificado de Luo et al., 2014). Una vez cocidos, se molieron y se extrajeron los compuestos siguiendo el mismo procedimiento que en las muestras crudas, donde también se calculó el rendimiento de extracto para cada muestra cocida. Posteriormente se resuspendieron en metanol hasta obtener una concentración de $0.178 \mathrm{~g}$ de extracto/mL. Las muestras se almacenaron a $-20{ }^{\circ} \mathrm{C}$ para su posterior análisis.

\section{Determinación de Fenoles totales}

Se realizó de acuerdo a la técnica de Folin Ciocalteu (Eldeen et al., 2011). Se tomaron $10 \mu \mathrm{L}$ de extracto y se agregaron $25 \mu \mathrm{L}$ de solución de Folin $1 \mathrm{~N}$. Se dejó reposar por 5 min a temperatura ambiente. Posteriormente se adicionaron $25 \mu \mathrm{L}$ de $\mathrm{Na}_{2} \mathrm{CO}_{3}$ al $20 \%$ y $140 \mu \mathrm{L}$ de agua destilada para llegar a un volumen final de $200 \mu \mathrm{L}$. Se dejó reposar 30 min y se determinó la absorbancia a $760 \mathrm{~nm}$. Se realizó una curva de calibración entre 0 y $1 \mathrm{mg} / \mathrm{mL}$ con el estándar ácido gálico, la calibración fue medida por triplicado. Los resultados se expresaron como miligramos de equivalentes de ácido gálico por gramo de muestra (mg EAG/g). Todas las mediciones se realizaron por triplicado.

\section{Determinación de Flavonoides}

Los flavonoides totales se realizaron por el método colorimétrico basado en Venu et al. (2012). Se tomaron 80 $\mu \mathrm{L}$ de extracto y fueron adicionadas a $80 \mu \mathrm{L}$ de una solución etanólica de tricloruro de aluminio ( $20 \mathrm{~g} / \mathrm{L}$ ). Se agitaron por 30 s y se cubrieron con parafilm. Se dejaron reposar durante $1 \mathrm{~h}$ en oscuridad a temperatura ambiente. Posteriormente se volvieron a agitar durante 30 s y se midió la absorbancia a 415 $\mathrm{nm}$. Se realizó una curva de calibración entre 0 y $0.8 \mathrm{mg} / \mathrm{mL}$ con el estándar querencitina, la calibración fue medida por triplicado. Los resultados se expresaron como miligramos de equivalentes de quercetina por gramo de muestra (mg EQ/g). 


\section{Capacidad antioxidante por ABTS (ácido 2,2'-azi- no-bis-(3 etilbenzotiazolin-6-sulfónico))}

La preparación del radical se realizó según la metodología de Re et al. (1999). Para la preparación del radical, se tomaron $19.3 \mathrm{mg}$ de ABTS y se disolvieron en $5 \mathrm{~mL}$ de agua destilada, por separado se pesaron $0.0378 \mathrm{~g}$ de persulfato de potasio y se mezclaron con $1 \mathrm{~mL}$ agua. Posteriormente se tomaron $88 \mu \mathrm{L}$ de la solución de persulfato y se agregaron a la solución de ABTS dejándose en oscuridad por 12 horas. De esta última solución se preparó una solución ajustada a una absorbancia de $0.7 \pm 0.01$ a una longitud de onda de 734 $\mathrm{nm}$. Se tomaron $270 \mu \mathrm{L}$ de la solución del radical catiónico preparado y se adicionaron $20 \mu \mathrm{L}$ de muestra leyendo a una absorbancia de $734 \mathrm{~nm}$ después de $30 \mathrm{~min}$ de reposo. Se realizó una curva de calibración entre 0 y $1 \mathrm{mg} / \mathrm{mL}$ con Trolox (Ácido-6-hidroxi-2,5,7,8-tetrametilcroman-2-carboxílico), la calibración fue medida por triplicado. Los resultados se expresaron en $\mu \mathrm{M}$ de equivalentes Trolox por gramo de

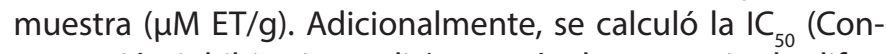
centración inhibitoria media) a través de una serie de diferentes concentraciones $(0,25,50,75,100,125,150,175$ у 200 $\mu \mathrm{g} / \mathrm{mL}$ ) calculando el porcentaje de inhibición de acuerdo a la siguiente ecuación:

\section{Capacidad antioxidante por DPPH (2,2-Difenil-1-Picrilhi- drazilo)}

Se pesaron $1.5 \mathrm{mg}$ de radical DPPH'y se disolvieron en $50 \mathrm{~mL}$ de metanol de los cuales se ajustó a una absorbancia de $0.7 \pm 0.01$ en una longitud de onda de $515 \mathrm{~nm}$ (Molyneux, 2004). De esta mezcla se tomaron $200 \mu \mathrm{L}$ de radical con 20 $\mu \mathrm{L}$ de muestra. Se dejó reposar $30 \mathrm{~min}$ en oscuridad y se midió a una longitud de onda de $515 \mathrm{~nm}$. Se realizó una curva estándar con trolox desde 0 a $200 \mu \mathrm{M}$. Los resultados se expresaron en $\mu \mathrm{M}$ de equivalentes Trolox por gramo de muestra $\left(\mu \mathrm{M} \mathrm{ET/g).} \mathrm{Adicionalmente,} \mathrm{se} \mathrm{calculó} \mathrm{la} \mathrm{IC}_{50}\right.$ (Concentración inhibitoria media) de la misma forma que con el radical $\mathrm{ABTS}^{++}$.

\section{Poder antioxidante reductor férrico (FRAP- Ferric redu- cing antioxidant power)}

El poder antioxidante por reducción del ión férrico a ferroso, se determinó utilizando la metodología de Rubio et al. (2016) con modificaciones. Primero se preparó una solución stock, la cual se realizó en condiciones ácidas $(\mathrm{pH}$ 3.6), que incluye una solución de buffer de acetato de sodio (300 mmol/L a un $\mathrm{pH}$ de 3.6). Posteriormente se preparó el complejo de hierro-TPTZ con $20 \mathrm{mmol}$ de $\mathrm{FeCl}_{3} \cdot 6 \mathrm{H}_{2} \mathrm{O}$ en una solución de TPTZ (2,4,6-tripiridil-s-triazin) en $40 \mathrm{mmol}$ de $\mathrm{HCl}$. Una vez preparadas las soluciones Stock, se procedió a preparar la solución de trabajo (solución de FRAP). Donde, se agregaron las soluciones a una relación de 10:1:1 (Buffer: $\mathrm{FeCl}_{3} \cdot 6 \mathrm{H}_{2} \mathrm{O}$ : TPTZ $\cdot \mathrm{HCl}$ ). Un volumen de $20 \mu \mathrm{L}$ de muestra se agregó a $280 \mu \mathrm{L}$ de la solución de FRAP, se tomaron lecturas a los 30 min en un lector de microplaca de 96 pocillos (Thermo Fisher Scientific Inc. Multiskan GO, NY, USA) a $638 \mathrm{~nm}$. La reducción del ión férrico ocurre visualmente en un cambio de color, desde transparente a azul. Se realizó una curva estándar con trolox, con concentraciones que van desde 0 a 200 $\mu \mathrm{M}$. Los resultados se expresaron como $\mu \mathrm{M} \mathrm{ET/g}$ de muestra.

\section{Sistema gastrointestinal in vitro}

La determinación de la digestibilidad y bioaccesibilidad para los extractos de cada muestra de frijol, se realizó a través de un modelo gastrointestinal in vitro de acuerdo con la metodología usada por Van-Campen y Glahn (1999) y Tarko et al. (2009). Consistió en la exposición de la harina de frijol cocida a enzimas digestivas: pepsina y pancreatina, evaluando el contenido de compuestos fenólicos, flavonoides y capacidad antioxidante. Se seleccionó a un voluntario aparentemente sano, el cual se lavó los dientes con pasta dental y el último bocado que probó fue a 90 min antes de la prueba. Se dieron $15 \mathrm{~g}$ de harina de frijol cocido a masticar 15 veces durante 15 segundos. Se homogenizó con $10 \mathrm{~mL}$ de agua purificada. Posteriormente se acidificaron las muestras con $\mathrm{HCl} 6 \mathrm{M}$ hasta llegar a un $\mathrm{pH}$ de 2 . Se adicionaron $22.5 \mathrm{~mL}$ de pepsina (315 U/mL) (Sigma, P7012-5G) y $22.5 \mathrm{~mL}$ de agua destilada. La muestra se colocó en un shaker con baño maría (modelo) a $80 \mathrm{rpm}$ a $37^{\circ} \mathrm{C}$ durante 2 horas. Una vez transcurrido este tiempo se neutralizaron las muestras $(\mathrm{pH} 7)$ con $\mathrm{NaHCO}_{3} 1.25 \mathrm{M}$ y se agregaron $5.625 \mathrm{~mL}$ de pancreatina (4 $\mathrm{mg} / \mathrm{mL}$ ) (Sigma, P1750-100G) a cada matraz. Se homogenizó bien y las muestras se colocaron dentro de baño María con shaker a $80 \mathrm{rpm}$ durante 4 horas a una temperatura de $37^{\circ} \mathrm{C}$. Posteriormente se realizaron las mediciones correspondientes (fenoles, flavonoides y capacidad antioxidante).

\section{Análisis estadístico}

Se realizó estadística descriptiva, análisis de varianza unifactorial con comparaciones múltiples de Tukey a un nivel de significancia de $5 \%(p<0.05)$, utilizando el paquete estadístico Infostat (versión 2008). Los resultados se expresaron como medias \pm desviación estándar de tres determinaciones.

\section{RESULTADOS Y DISCUSION}

\section{Rendimiento de los extractos}

El rendimiento de los extractos crudo y cocido obtenidos de cada una de las muestras de frijol se observan en la (Tabla 1). La variedad Pinto Santillo, obtuvo el mayor rendimiento del extracto de frijol crudo. Así como, un decremento en el rendimiento del extracto cocido en las variedades de frijol negro, Flor de Mayo y Pinto Saltillo. Sin embargo, las variedades de frijol Teapa y Peruano incrementaron 3.4 y 1.57 veces respectivamente respecto al crudo, mientras que el frijol Patol prácticamente no se vio afectado.

Al respecto, distintos autores como Madhujith y Shahidi (2005), han reportado trabajos de frijol crudo, donde el frijol negro presentó el mayor rendimiento de extracto $(0.206 \mathrm{~g}$ de extracto $/ \mathrm{g})$, frijol rojo $(0.167 \mathrm{~g}$ de extracto $/ \mathrm{g})$, frijol blanco ( $0.141 \mathrm{~g}$ de extracto $/ \mathrm{g})$ y frijol marrón $(0.171 \mathrm{~g}$ de extracto $/ \mathrm{g})$. Si se compara con nuestro estudio, el rendimiento de frijol negro es menor, esta diferencia puede deberse al tipo de solvente utilizado, ya que en nuestro estudio se utilizó metanol 
Tabla 1. Rendimiento de extractos obtenidos de muestras de frijol antes y después del cocimiento.

Table 1. Extract yields obtained from bean samples before and after cooking.

\begin{tabular}{lcccc}
\hline & & & \multicolumn{2}{c}{ Rendimiento } \\
\cline { 4 - 5 } Muestra frijol & $\begin{array}{c}\text { Peso inicial } \\
\text { (g) }\end{array}$ & $\begin{array}{c}\text { Peso de } \\
\text { extracto }(\mathbf{g})\end{array}$ & $\begin{array}{c}\text { g extracto/g } \\
\text { muestra }\end{array}$ & $\%$ \\
\hline Crudo & & & & \\
\hline Negro & $15.04 \pm 0.01$ & $1.92 \pm 0.22$ & $0.13 \pm 0.011^{\mathrm{b}}$ & $13 \pm 1.1^{\mathrm{b}}$ \\
\hline Flor de mayo & $15.02 \pm 0.02$ & $1.86 \pm 0.14$ & $0.12 \pm 0.003^{\mathrm{b}}$ & $12 \pm 0.3^{\mathrm{b}}$ \\
\hline Patol & $15.07 \pm 0.01$ & $1.15 \pm 0.31$ & $0.08 \pm 0.001^{\mathrm{c}}$ & $8 \pm 0.1^{\mathrm{c}}$ \\
\hline Pinto Saltillo & $15.05 \pm 0.01$ & $3.69 \pm 0.28$ & $0.25 \pm 0.013^{\mathrm{a}}$ & $25 \pm 1.3^{\mathrm{a}}$ \\
\hline Teapa & $15.07 \pm 0.02$ & $0.78 \pm 0.03$ & $0.05 \pm 0.002^{\mathrm{d}}$ & $5 \pm 0.2^{\mathrm{d}}$ \\
Peruano & $15.05 \pm 0.03$ & $1.10 \pm 0.09$ & $0.07 \pm 0.003^{\mathrm{c}}$ & $7 \pm 0.3^{\mathrm{c}}$ \\
\hline Cocido & & & & \\
\hline Negro & $15.17 \pm 0.10$ & $0.53 \pm 0.01$ & $0.03 \pm 0.001^{\mathrm{d}}$ & $3 \pm 0.1^{\mathrm{d}}$ \\
\hline Flor de mayo & $15.03 \pm 0.01$ & $1.54 \pm 0.25$ & $0.10 \pm 0.001^{\mathrm{b}}$ & $10 \pm 0.1^{\mathrm{b}}$ \\
\hline Patol & $15.22 \pm 0.15$ & $1.49 \pm 0.08$ & $0.09 \pm 0.002^{\mathrm{bc}}$ & $9 \pm 0.2^{\mathrm{bc}}$ \\
\hline Pinto Saltillo & $15.52 \pm 0.34$ & $1.08 \pm 0.12$ & $0.07 \pm 0.001^{\mathrm{c}}$ & $7 \pm 0.1^{\mathrm{c}}$ \\
\hline Teapa & $15.09 \pm 0.03$ & $2.59 \pm 0.09$ & $0.17 \pm 0.031^{\mathrm{a}}$ & $17 \pm 3.1^{\mathrm{a}}$ \\
\hline Peruano & $15.15 \pm 0.11$ & $1.66 \pm 0.05$ & $0.11 \pm 0.022^{\mathrm{b}}$ & $11 \pm 2.2^{\mathrm{b}}$ \\
\hline
\end{tabular}

Media \pm Desviación estándar /Mean \pm standard deviation. Letras diferentes en la misma columna indica diferencia estadística significativa $(p<0.05)$ para cada tratamiento.

al $80 \%$ para la extracción y en el de los otros autores fueron extraídos con acetona al $80 \%$. Por lo tanto, resulta complicado comparar rendimientos de extractos de los diferentes estudios en frijol por todas las variaciones implícitas en los procesos de extracción. Por otra parte, se puede observar que existen diferencias en los rendimientos de extracto entre las distintas variedades. Es probable que estas diferencias se deban a los tipos de compuestos que conforman a los extractos, ya que cada variedad de frijol puede verse afectada en su composición por varios factores como al genotipo, altitud, temperatura, humedad y suelo de su lugar de procedencia (Aleum, 2017).

Uno de los beneficios del cocimiento del frijol es la de mejorar sus propiedades físicas y organolépticas, además, de que incrementa el uso biológico de las proteínas, debido a que se destruyen ciertos factores termolábiles como son los inhibidores de funciones metabólicas, tripsina, hemaglutinina y otros inhibidores enzimáticos (García, 2014). De ahí la importancia de consumir el frijol cocido y de analizarlo también de esta forma en nuestro estudio. Sin embargo, el efecto del cocimiento se ve reflejado en algunas de las muestras decreciendo o bien aumentando su rendimiento. Se sabe que el cocimiento puede llegar a degradar los compuestos influyendo en el rendimiento final. Por otra parte, puede haber una transformación de ellos y/o formación de nuevos compuestos ya que la temperatura afecta a las estructuras formando nuevas moléculas, lo cual podría explicar el incremento en los rendimientos del frijol Teapa y Peruano.

\section{Contenido Fenólico}

Se determinó el contenido de fenoles en las diferentes variedades de frijol antes y después del cocimiento (Figura

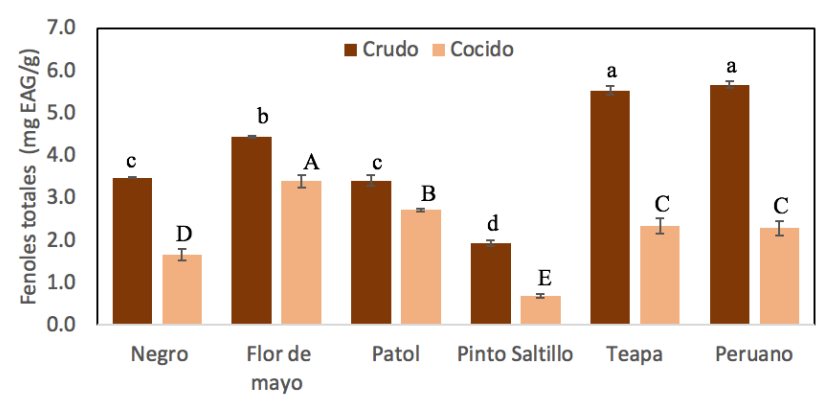

Figura 2. Cuantificación de fenoles totales de las diferentes muestras de frijol antes y después del cocimiento. Letras diferentes indican diferencia estadística significativa $(p<0.05)$. Letras minúsculas se comparan entre los tipos de frijol crudo y letras mayúsculas entre los tipos de frijol cocido.

Figure 2. Total phenols quantification of the different bean samples before and after cooking. Different letters indicate statistically significant difference $(p<0.05)$.

2). Los valores obtenidos en nuestro estudio oscilan entre 1.91 a $5.66 \mathrm{mg} \mathrm{EAG/g}$ en frijol crudo y valores de 0.68 a 3.47 mg EAG/g en frijol cocido. Las variedades de frijol Teapa y Peruano son las que presentaron mayor cantidad de fenoles totales antes del cocimiento. Sin embargo, después del cocinado disminuyen más de la mitad. Las variedades Flor de Mayo y Patol son las que conservan la mayor cantidad de compuestos fenólicos después de haberlos sometido a cocimiento.

Algunas investigaciones han mencionado la relación del color del frijol con el contenido de compuestos fenólicos, en donde los frijoles con color claro contienen menor cantidad de fenoles en comparación a los frijoles oscuros (Xu et al., 2009). Sin embargo, en el presente estudio el frijol peruano con pigmentación amarilla presentó el valor más alto en fenólicos a diferencia del frijol negro. Así mismo, autores como Rocha-Guzmán et al. (2007) han encontrado que el frijol cocido disminuye de 73 a $90 \%$ la cantidad de contenido fenólico por efecto de lixiviación, en donde a mayor temperatura mayor lixiviación. Huber et al. (2016) mencionan que el cocimiento aumenta la concentración del contenido fenólico. La Farga et al. (2019) argumenta que el aumento se debe a una mayor extracción causado por la ruptura celular durante la cocción. Estas diferencias pueden atribuirse al procesamiento térmico, remojo, condiciones de almacenamiento, ambientales y genotipos.

\section{Flavonoides}

Se determinó el contenido de flavonoides en las diferentes variedades de frijol antes y después del cocimiento (Figura 3). Los valores obtenidos en nuestro estudio oscilan entre 0.83 a $3.31 \mathrm{mg} \mathrm{EQ/g}$ en frijol crudo y valores de 0.21 a $3.70 \mathrm{mg} \mathrm{EQ} / \mathrm{g}$ en frijol cocido, cantidades más bajas a los fenoles debido a que son un subgrupo de compuestos fenólicos. Sin embargo, las variedades de frijol crudo Flor de Mayo y Patol presentaron la mayor cantidad de flavonoides, pero también son los que presentan mayor disminución de los mismos después del cocimiento. En cambio, las variedades Teapa, Peruano y Pinto Saltillo, mostraron un incremento 


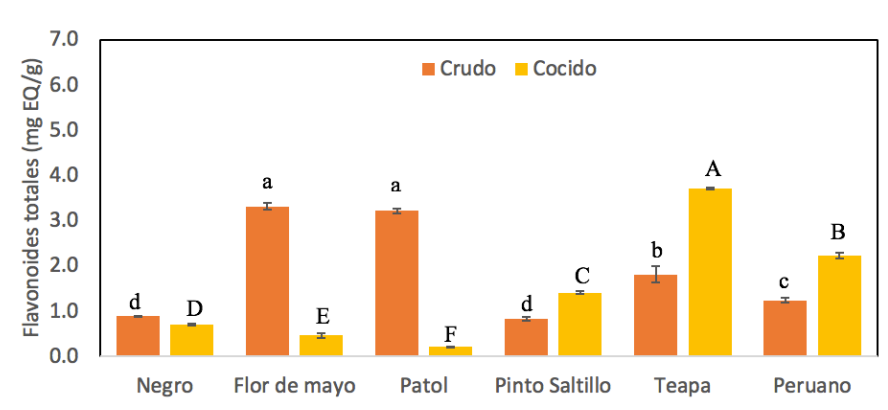

Figura 3. Cuantificación de flavonoides totales de las diferentes muestras de frijol antes y después del cocimiento. Letras diferentes indican diferencia estadística significativa $(p<0.05)$.

Figure 3. Total flavonoids quantification of the different bean samples before and after cooking.

Different letters indicate statistically significant difference $(p<0.05)$.

de flavonoides después del cocinado. Huber et al. (2016) menciona que cuando el frijol se somete a cocimiento se presenta un aumento de flavonoides. Contrariamente, $\mathrm{Xu}$ et al. (2009), reportan que remojar y cocinar el frijol ejerce un efecto negativo en las concentraciones de flavonoides. Por lo que en nuestro estudio el procesamiento de cocción causó ambos efectos en las distintas variedades de frijol.

\section{Capacidad Antioxidante}

Los resultados obtenidos en capacidad antioxidante por DPPH, ABTS y FRAP en las diferentes variedades de frijol antes y después del cocimiento se pueden observar en la Figura 4. Los valores obtenidos en nuestro estudio oscilan entre 0.60 a $3.01 \mu \mathrm{M} \mathrm{ET/g}$ en frijol crudo y valores de 0.58 a $2.90 \mu \mathrm{M}$ ET/g en frijol cocido en DPPH; 0.68 a $2.18 \mu \mathrm{M} \mathrm{ET/g} \mathrm{en}$ frijol crudo y valores de 0.52 a $1.94 \mu \mathrm{M} \mathrm{ET/g}$ en frijol cocido en ABTS; y 5.24 a $36.99 \mu \mathrm{M} \mathrm{ET/g}$ en frijol crudo y valores de 5.22 a $17.80 \mathrm{mg}$ EAG/g en frijol cocido en FRAP. La cocción incrementó la actividad antioxidante del radical DPPH en las variedades de frijol Negro y Patol. Sin embargo, disminuyó en las variedades de Flor de Mayo y Pinto Saltillo. En Teapa se mantuvo estable. En ABTS las variedades de Negro, Flor de Mayo, Teapa y Peruano observaron un incremento de la actividad antioxidante después del cocimiento, mientras que en Patol y Pinto Saltillo decreció. Para FRAP casi todos estuvieron estables con ligero aumento en Negro y Peruano, y ligera disminución en Flor de mayo, Patol y Pinto Saltillo. Solo la variedad Teapa presentó una disminución considerable del $52 \%$ después del cocimiento.

La combinación de estos ensayos proporcionó una mayor evaluación de las actividades antioxidantes de las muestras de frijol antes y después del cocimiento. Además, autores como Xu y Chang (2009), Gálvez et al. (2009), Rocha-Guzmán et al. (2009) y Huber et al. (2016) evaluaron la capacidad antioxidante de variedades de frijol Negro, Pinto Satillo, Flor de Mayo, antes y después de cocción en remojo y sin remojo y presentan comportamientos similares a los obtenidos en este estudio. Botaeng et al. (2007) menciona que la cocción y retención de los componentes bioactivos después del cocimiento, presenta cantidades adecuadas de antioxidantes para una dieta saludable. Sin embargo,

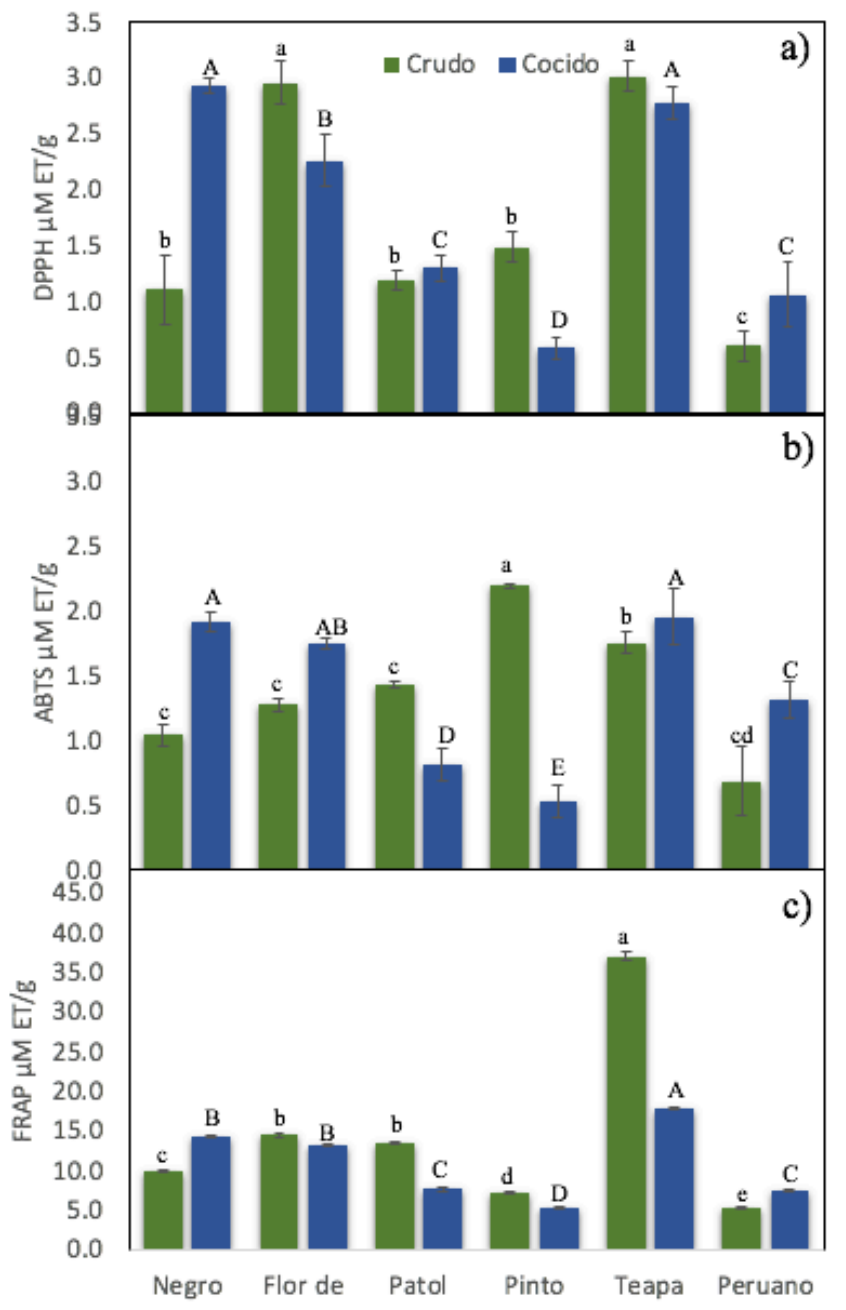

Figura 4. Capacidad antioxidante por DPPH (a), ABTS (b) Y FRAP (c) de las diferentes muestras de frijol antes y después del cocimiento. Letras diferentes indican diferencia estadística significativa $(p<0.05)$.

Figure 4. Antioxidant capacity by DPPH (a), ABTS (b) and FRAP (c) of the different bean samples before and after cooking. Different letters indicate statistically significant difference $(p<0.05)$.

la forma de preparación de los alimentos puede ser tan importante como el alimento en sí. Por lo tanto, aún con la disminución de bioactivos después de la cocción existe actividad antioxidante, por lo que una dieta rica en leguminosas podría beneficiar a la salud humana.

En la figura 5 se observa la cinética de inhibición al radical DPPH para determinar la concentración inhibitoria media $\left(\mathrm{IC}_{50}\right)$ de los extractos de frijol, en donde las variedades Teapa con $106.45 \mu \mathrm{g} / \mathrm{mL}$ y Flor de Mayo con $127.16 \mu \mathrm{g} / \mathrm{mL}$, fueron las que alcanzaron el $50 \%$ de inhibición en las concentraciones estudiadas. En ABTS (Figura 5), las variedades de Teapa con $176.21 \mu \mathrm{g} / \mathrm{mL}$ y Pinto Saltillo $154.72 \mu \mathrm{g} / \mathrm{mL}$, fueron las muestras que lograron alcanzar la $\mathrm{IC}_{50}$ en este radical. La composición de los extractos juega un papel importante para poseer capacidad antioxidante. Generalmente con el método de extracción que se utilizó en este estudio, se obtienen compuestos fenólicos. De todos los compuestos fenólicos, el grupo de los flavonoides es el más extendido en la naturaleza y dentro de ellos, los flavonoles son los que 


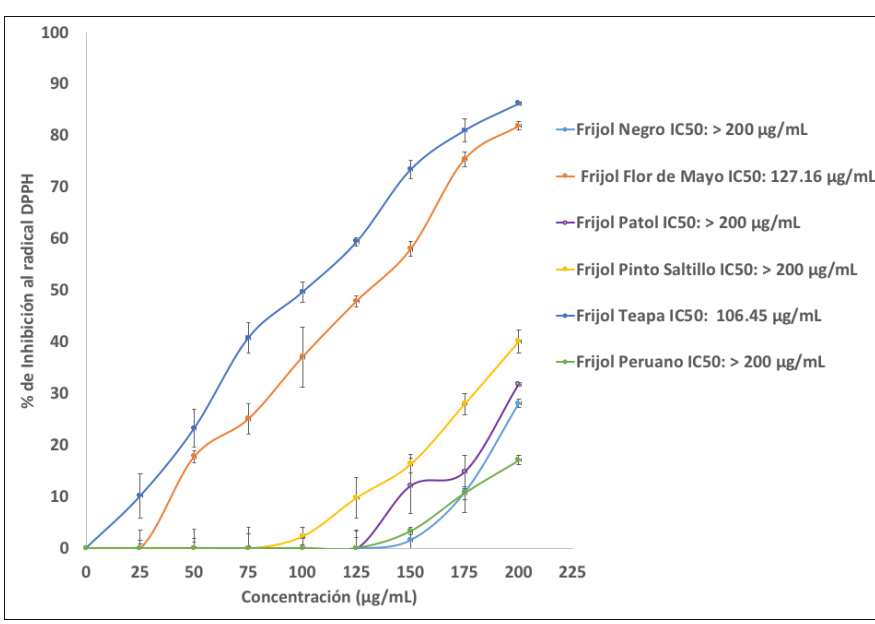

Figura 5. Cinéticas de inhibición del radical DPPH para la determinación de las concentraciones inhibitorias medias $\left(\mathrm{IC}_{50}\right)$ de los extractos de frijol. Figure 5. Inhibition kinetics of DPPH radical to determine the medium inhibitory concentrations $\left(\mathrm{IC}_{50}\right)$ from bean extracts.

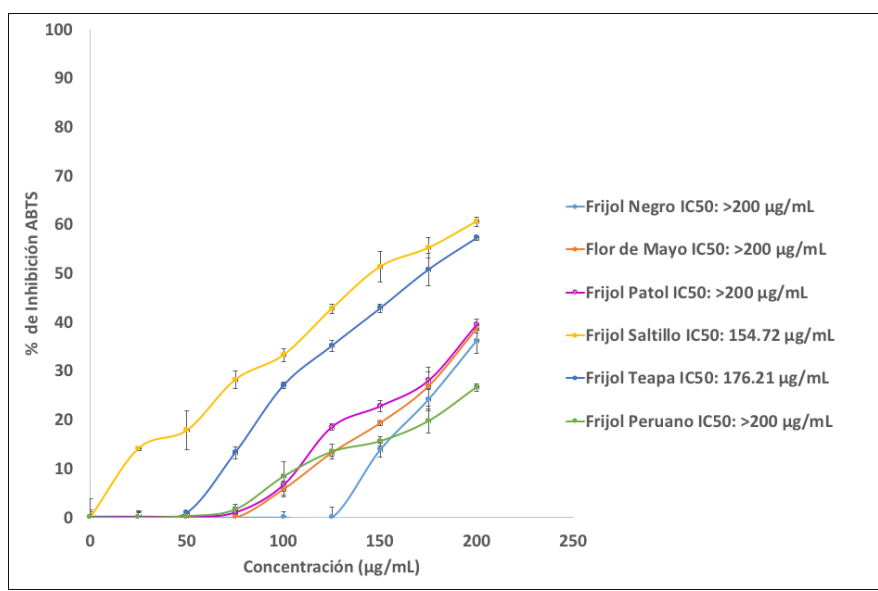

Figura 6. Cinéticas de inhibición del radical ABTS para la determinación de las concentraciones inhibitorias medias $\left(\mathrm{IC}_{50}\right)$ de los extractos de frijol. Figure 6. Inhibition kinetics of ABTS radical to determine the medium inhibitory concentrations $\left(\mathrm{IC}_{50}\right)$ from bean extracts.

poseen un mayor potencial antioxidante (Perez-Perez et al., 2018). Sin embargo, para poder explicar si estos compuestos confieren realmente la mayor capacidad antioxidante, se realizaron correlaciones (Tabla 2).

De acuerdo a las correlaciones realizadas con las diferentes muestras de frijol, los flavonoides son los principales componentes de los compuestos fenólicos ya que en cada muestra la correlación de fenoles con flavonoides fue muy alta $\left(R^{2}>0.96\right)$. Estos compuestos parecen ser que son los responsables de conferir la capacidad antioxidante y/o poder reductor en frijol Negro (FRAP), Flor de mayor (DPPH y FRAP), Patol (ABTS y FRAP), Pinto Saltillo (ABTS y FRAP), Teapa (DPPH y ABTS) y Peruano (ABTS y FRAP). Al parecer, los compuestos que conforman a los diferentes extractos de frijol son más afines al radical ABTS que al DPPH, y confieren mayor poder reductor en FRAP. Estas diferencias pueden deberse a que el radical $A B T S$ puede reaccionar con compuestos aromáticos hidroxilados en un amplio rango de $\mathrm{pH}$, factor que limita al radical DPPH ya que trabaja con $\mathrm{pH}$ neutros o alcalinos (Prior
Tabla 2. Correlaciones $\left(\mathrm{R}^{2}\right)$ de la capacidad antioxidante contra los fenoles y flavonoides de los extractos de frijol.

Table 2. Correlations $\left(\mathrm{R}^{2}\right)$ of the antioxidant capacity versus phenols and flavonoids of bean extracts.

\begin{tabular}{|c|c|c|}
\hline & \multicolumn{2}{|c|}{ Correlaciones $\left(\mathbf{R}^{2}\right)$} \\
\hline & Fenoles & Flavonoides \\
\hline \multicolumn{3}{|c|}{ Frijol Negro } \\
\hline DPPH & 0.4541 & 0.5749 \\
\hline ABTS & 0.6131 & 0.7617 \\
\hline FRAP & 0.9922 & 0.982 \\
\hline Fenoles & - & 0.9686 \\
\hline \multicolumn{3}{|c|}{ Frijol Flor de Mayo } \\
\hline DPPH & 0.9917 & 0.9872 \\
\hline ABTS & 0.6676 & 0.6784 \\
\hline FRAP & 0.996 & 0.9987 \\
\hline Fenoles & - & 0.9978 \\
\hline \multicolumn{3}{|l|}{ Frijol Patol } \\
\hline DPPH & 0.5829 & 0.6433 \\
\hline ABTS & 0.8874 & 0.8874 \\
\hline FRAP & 1 & 1 \\
\hline Fenoles & - & 1 \\
\hline \multicolumn{3}{|c|}{ Frijol Pinto Saltillo } \\
\hline DPPH & 0.796 & 0.7901 \\
\hline ABTS & 0.9863 & 0.9767 \\
\hline FRAP & 1 & 0.9875 \\
\hline Fenoles & - & 0.9875 \\
\hline \multicolumn{3}{|c|}{ Frijol Teapa } \\
\hline DPPH & 0.9888 & 0.9832 \\
\hline ABTS & 0.9654 & 0.9615 \\
\hline FRAP & 0.5289 & 0.5035 \\
\hline Fenoles & - & 0.9915 \\
\hline \multicolumn{3}{|c|}{ Frijol Peruano } \\
\hline DPPH & 0.6407 & 0.6163 \\
\hline ABTS & 0.9298 & 0.9267 \\
\hline FRAP & 0.9428 & 0.9826 \\
\hline Fenoles & & 0.9852 \\
\hline
\end{tabular}

et al., 2005; Roginsky y Lissi, 2005). Por otra parte, ambos radicales pueden ser donadores de $\mathrm{H}^{+}$pero el DPPH no reacciona con ácidos aromáticos que contienen solo un grupo $\mathrm{OH}$ ni tampoco con fenoles que no contengan un $\mathrm{OH}$ en el $\beta$-anillo (Yokosawa et al., 1998). Sin embargo, para poder confirmar lo anterior, se deberán identificar por HPLC los compuestos individuales de cada muestra.

\section{Sistema de simulación in vitro del sistema gastrointesti- nal}

En la tabla 3, se observa el contenido fenólico y actividad antioxidante de las diferentes variedades de frijol cocido y a través de un proceso de digestión in vitro. Los fenoles presentaron un incremento en todas las muestras que van de un rango de 1.14 a $4.62 \mathrm{mgEAG} / \mathrm{g}$, siendo la variedad Teapa la de mayor incremento. Esto pudo deberse a que el 
Tabla 3. Contenido fenólico y actividad antioxidante de diferentes variedades de frijol cocido a través de un proceso de digestión in vitro.

Table 3. Phenolic content and antioxidant activity of different varieties of cooked beans through an in vitro digestion process.

\begin{tabular}{|c|c|c|c|c|c|c|}
\hline & Negro & Flor de Mayo & Patol & Pinto Saltillo & Teapa & Peruano \\
\hline \multicolumn{7}{|l|}{$\begin{array}{l}\text { Fenoles } \\
\text { (mg EAG/g) }\end{array}$} \\
\hline Antes de digestión & $1.66 \pm 0.13^{c}$ & $3.39 \pm 0.15 a$ & $2.71 \pm 0.03^{b}$ & $0.68 \pm 0.04^{d}$ & $2.33 \pm 0.18^{b}$ & $2.28 \pm 0.16^{b}$ \\
\hline $\begin{array}{l}\text { Después de digestión } \\
\text { (Bioaccesibles) }\end{array}$ & $2.72 \pm 0.16^{d}$ & $3.49 \pm 0.10^{b}$ & $2.96 \pm 0.07^{c}$ & $2.52 \pm 0.10^{c}$ & $4.62 \pm 0.11^{\mathrm{a}}$ & $1.14 \pm 0.08^{e}$ \\
\hline \multicolumn{7}{|l|}{$\begin{array}{l}\text { Flavonoides } \\
\text { (mg ET/g) }\end{array}$} \\
\hline Antes de digestión & $0.70 \pm 0.038^{d}$ & $0.47 \pm 0.051^{\mathrm{e}}$ & $0.21 \pm 0.019^{f}$ & $1.41 \pm 0.046^{c}$ & $3.71 \pm 0.022^{\mathrm{a}}$ & $2.22 \pm 0.064^{b}$ \\
\hline $\begin{array}{l}\text { Después de digestión } \\
\text { (Bioaccesibles) }\end{array}$ & $0.32 \pm 0.004^{d}$ & $0.37 \pm 0.007^{d}$ & $0.03 \pm 0.002^{\mathrm{e}}$ & $0.92 \pm 0.005^{b}$ & $1.65 \pm 0.003^{\mathrm{a}}$ & $0.82 \pm 0.001^{c}$ \\
\hline \multicolumn{7}{|l|}{$\begin{array}{l}\text { DPPH } \\
(\mu \mathrm{mol} E T / g)\end{array}$} \\
\hline Antes de digestión & $2.92 \pm 0.06^{\mathrm{a}}$ & $2.25 \pm 0.23^{a}$ & $1.29 \pm 0.12^{b}$ & $0.58 \pm 0.09^{c}$ & $2.77 \pm 0.14^{\mathrm{a}}$ & $1.05 \pm 0.29^{b}$ \\
\hline Después de digestión & $1.50 \pm 0.03^{b}$ & $1.97 \pm 0.04^{\mathrm{a}}$ & $0.15 \pm 0.10^{b}$ & $0.33 \pm 0.12^{c}$ & $1.57 \pm 0.01^{b}$ & $0.28 \pm 0.13^{d}$ \\
\hline \multicolumn{7}{|l|}{$\begin{array}{l}\text { ABTS } \\
(\mu \mathrm{mol} E T / g)\end{array}$} \\
\hline Antes de digestión & $1.91 \pm 0.08^{\mathrm{a}}$ & $1.74 \pm 0.03^{b}$ & $0.81 \pm 0.12^{d}$ & $0.52 \pm 0.12^{\mathrm{e}}$ & $1.94 \pm 0.21^{\mathrm{a}}$ & $1.30 \pm 0.14^{c}$ \\
\hline Después de digestión & $0.43 \pm 0.06^{c}$ & $1.37 \pm 0.08^{\mathrm{a}}$ & $0.23 \pm 0.14^{\mathrm{e}}$ & $0.46 \pm 0.03^{c}$ & $1.21 \pm 0.03^{b}$ & $0.33 \pm 0.14^{d}$ \\
\hline \multicolumn{7}{|l|}{$\begin{array}{l}\text { FRAP } \\
(\mu \mathrm{mol} \mathrm{ET} / \mathrm{g})\end{array}$} \\
\hline Antes de digestión & $14.18 \pm 0.12^{b}$ & $13.02 \pm 0.11^{c}$ & $7.58 \pm 0.20^{d}$ & $5.22 \pm 0.04^{\mathrm{e}}$ & $17.8 \pm 0.09^{a}$ & $7.39 \pm 0.07^{d}$ \\
\hline Después de digestión & $10.45 \pm 0.05^{b}$ & $9.53 \pm 1.12^{b}$ & $6.1 \pm 0.77^{c}$ & $5.87 \pm 0.11^{d}$ & $13.21 \pm 1.3^{\mathrm{a}}$ & $5.21 \pm 0.92^{d}$ \\
\hline
\end{tabular}

Media \pm Desviación estándar /Mean \pm standard deviation. Letras diferentes en la misma fila indica diferencia estadística significativa $(p<0.05)$.

proceso de digestión consiguió liberar o dejar más libres a los compuestos fenólicos que están dentro de la matriz alimentaria, por lo tanto están más bioaccesibles. Sin embargo, con los flavonoides pasa lo contrario, parece ser que se degradan o bien se pueden transformar en compuestos con menos actividad antioxidante. Por lo que en este estudio y en otros similares en los que el frijol ha sido remojado, se presenta que el menor nivel de biodisponibilidad de los compuestos se debe a que después del remojo se lleva a cabo la liberación de compuestos fenólicos en el agua en que fueron remojados. Así mismo, Yuwei et al. (2014) presentan valores de frijol sin remojar de 3.24 y $4.86 \mathrm{mg} \mathrm{GAE} / \mathrm{g}$ en fenoles y valores de 0.24 a $1.38 \mathrm{mgCE} / \mathrm{g}$ muy parecidos a los obtenidos en este estudio. Autores como Surangi et al. (2013). mencionan que la biodisponibilidad de los flavonoides es relativamente baja debido a la absorción limitada y rápida eliminación. Más aún, los flavonoides son rápidamente metabolizados y las actividades biológicas de sus metabolitos no son siempre las mismas que las de sus predecesores. Por lo que, la biodisponibilidad de los flavonoides aún no se encuentra bien establecida.

Scalbert y Williamson (2000), indican que existen pocos datos y que una pequeña parte del compuesto es absorbido y como tal encontrado en plasma y orina entre el $7 \%$ y el $25 \%$ del compuesto ingerido. En cambio, en el plasma humano las concentraciones encontradas después de la ingesta son del orden de $1 \mu \mathrm{M}$ y decrecen. En nuestro estudio en comparación con el frijol cocido el $78 \%, 65 \%$, $44 \%$ y $45 \%$ del total de flavonoides fue bioaccesible en el sistema pancreático simulado, para las variedades de Frijol Negro, Flor de Mayo, Pinto Saltillo y Teapa, respectivamente. Sin embargo, la disminución de los flavonoides afectó tanto la capacidad antioxidante por DPPH y ABTS como para el poder reductor en FRAP ya que después del proceso de digestión mostraron un disminución de actividad antioxidante, quedando la mayor actividad en DPPH del $87 \%, 57 \%$ y $57 \%$ para Flor de Mayo, Pinto Saltillo y Teapa; en ABTS 79\% 88\% y $62 \%$ para las mismas variedades. En el poder reductor FRAP hay un incremento del $8.82 \%$ en Pinto Saltillo. Estos valores sugieren cambios importantes en los compuestos fenólicos y flavonoides como modificaciones de la estructura química, aumento o disminución de la solubilidad o de la interacción con otros compuestos durante la digestión de las muestras utilizadas, que pueden influenciar en la bioaccesibilidad (Soriano et al., 2015). Sin embargo, a pesar de que hubo una disminución en la capacidad antioxidante, la actividad no es 
tan baja y podría tener un efecto benéfico en la prevención de enfermedades crónico degenerativas a través de la inhibición de los radicales libres.

Finalmente, aunque hay varios estudios que reportan la bioaccesibilidad y biodisponibilidad de estos componentes de los alimentos, en un intento de entender sus interacciones, debe considerarse que los actuales métodos analíticos para estimarlos en los seres humanos a través de la identificación de los principales metabolitos tienen sus limitaciones. Por lo que hacen falta más estudios para poder explicar a más detalle estos comportamientos.

\section{CONCLUSIONES}

De las seis variedades evaluadas de frijol, las muestras Teapa, Pinto Saltillo y Flor de Mayo presentaron un incremento del contenido fenólico, flavonoides, y actividad antioxidante, observándose un efecto positivo al cocimiento. Así mismo, el proceso de digestión in vitro mostró una bioaccesibilidad mayor para compuestos fenólicos. Lo anterior repercutió en una disminución de la capacidad antioxidante, pero a pesar de que hubo esta disminución, la actividad no es tan baja y podría tener un efecto benéfico en la prevención de enfermedades a través de la inhibición de los radicales libres. Sin embargo, se requieren análisis adicionales para profundizar un poco más sobre este estudio, primeramente, determinando los compuestos individuales de cada extracto y posteriormente evaluando cuál de ellos confiere mayor capacidad antioxidante a las muestras después del proceso de digestión.

\section{AGRADECIMIENTOS}

Al consejo de Ciencia y Tecnología CONACYT México por el apoyo otorgado en la Convocatoria Proyecto de Desarrollo Científico para atender Problemas Nacionales 2015, al Proyecto 1529 "Biofortificación de cultivos agrícolas básicos, clave para combatir la desnutrición y seguridad alimentaria en México". A la Facultad de Ciencias Agrotecnológicas de la Universidad Autónoma de Chihuahua, por el apoyo brindado para la realización de una estancia de investigación en la Universidad de Sonora resultando este artículo científico.

\section{REFERENCIAS}

Aleum, H. 2017. Review paper on breeding common bean (Phaseolus vulgaris L.) genotypes for acidic soil tolerance. International Journal of Advanced research and publications. $13)$ 39-46.

Boateng, J.V.M. 2008. Effect of processing on antioxidant contents in selected dry beans (Phaseolus spp. L.). Food Science and Technology. 41: 1541-1547.

Cardona, F., Andrés-Lacueva, C., Tulipania, S., Tinahonesb, F.J., Queipo-Ortuñoa, M.I. 2013. Benefits of polyphenols on gut microbiota and implications in human health. Sciverse ScienceDirect. 24: 1415-1422.

Celmeli, T, Sari, H, Canci, H, Sari, D, Adak, A, Eker, T, Toker, C. 2018. The nutritional content of common bean (Phaseolus vulgaris L.) Landraces in comparison to modern varieties. Agronomy.
8(166): 1-9.

Chia-Jung, Ch., Kadouh, H., Kequan, Z. 2013. Phenolic compounds and antioxidant properties of gooseberry as affected by in vitro digestion. Food Science and Technology. 51: 417-422.

Díaz, A.M., Caldas, G.V., y Blair, M.W. 2010. Concentrations of condensed tannins and anthocyanins in common bean seed coats. Food Research International. 43(2): 595-601.

Eldeen, I.M.S., Seow, E.M., Abdullah, R., Sulaiman, S.F. 2011. In vitro antibacterial, antioxidant, total phenolic contents and anti-HIV-1 reverse transcriptase activities of extracts of seven Phyllanthus sp. South African Journal of Botany. 77: 75-79.

Gálvez, J., Coelho, G., Crespo, M.E., Cruz, T., Rodríguez-Cabezas, M.E., Concha, A. 2001. Intestinal anti-inflammatory activity of morin on chronic experimental colitis in the rat. Alimentary Pharmacology \& Therapeutics. 15: 2027-2039.

Gálvez, R.L, Genovese, M.A., Lajolo, F.M. 2009. Effect of Different Cooking Conditions on Phenolic Compounds and Antioxidant Capacity of Some Selected Brazilian Bean (Phaseolus vulgaris L.) Cultivars. J. Agric. Food Chem. 57: 5734-5742.

García, E.C. 2014. Inhibidores de Proteasas en Leguminosas. Tesis de Licenciatura. Universidad de Valladolid. 29 p.

Gutiérrez-Grijalva, E.P., Ambriz-Pérez, D.L., Leyva-López, N., Castillo-López, R.I. y Heredia, J.B. 2016. Biodisponibilidad de compuestos fenólicos dietéticos: Revisión. Revista Española de Nutrición Humana y Dietética. 20(2): 140-147.

Huber, K. 2016. Phenolic Acid, Flavonoids and Antioxidant Activity of Common Brown Beans (Phaseolus vulgaris L.) Before and After Cooking. Journal of Nutrition \& Food Sciences. 6(5): 1-7.

Kähkönen, M. P., Hopia, A. I., y Heinonen, M. 2001. Berry phenolics and their antioxidant activity. Journal of Agricultural and Food Chemistry. 49: 4076e4082.

Kahlon, T. S., y Smith, G. E. 2007. In vitro binding of bile acids by blueberries (Vaccinium spp.), plums (Prunus spp.), prunes (Prunus spp.), strawberries (Fragaria X ananassa), cherries (Malpighia punicifolia), cranberries (Vaccinium macrocarpon) and apples (Malus sylvestris). Food Chemistry. 100: 1182e1187.

Karas,_M., Jakubczyk, A., Szymanowska, U., Złotek, U., y Zieli_nska, E. 2016. Digestion and bioavailability of bioactive phytochemicals. International Journal of Food Science and Technology. 1-5.

La Farga, T., Villaro, S., Bobo, G., Simo, J., Agullo-Aguallo, I. 2019. Bioaccessibility and antioxidant activity of phenolic compounds in cooked pulses. Food Science Technology. 1-8.

Laparra, J.M., Glahn, R. P., Miller, D.D. 2008. Bioaccessibility of phenols in common beans (Phaseolus vulgaris L.) and iron (Fe) availability to Caco-2 Cells. J. Agric. Food Chem. 56, 10999-11005.

Luo, Y., Xie, W., Hao, Z., Jin, X., Wang, Q. 2014. The impact of processing on in vitro bioactive compounds bioavailability and antioxidant activities in faba bean (Vicia faba L.) and azuki bean (Vigna angularis L.). International Food Research Journal. 21(3): 995-1001.

Maarit, J.R., Renouf, M., Cruz-Hernandez, C., Actis-Goretta, M., Sagar K., y Da Silva M. 2013. Bioavailability of bioactive food compounds: a challenging journey to bioefficacy. Br. J. Clin Pharmacol. 75(3): 588-602. 
Madhujith, T., Shahidi, F. 2005. Antioxidant potential of pea beans (Phaseolus vulgaris L). Journal of Food Science. 70(1): S85-S90.

Manach, C., Scalbert, A., Morand, C., Rémésy, C., Jimenez, L. 2004. Polyphenols: food sources and bioavailability Am. J. Clin Nutr. 79: 727-747.

Mojica, L., Chen, K., González de Mejía, E. 2014. Impact of Commercial Precooking of common bean (Phaseolus vulgaris) on the generation of peptides, after pepsinpancreatin hydrolysis, capable to inhibit dipeptidyl peptidase-IV. 80(1): 188-198.

Molyneux, P. 2004. The use of the stable radical dipheylpicrylhydrazyl (DPPH) for estimating antioxidant activity. Songklanakarin Journal of Science Technology, 26(2): 211-219.

Pérez-Perez L.M., García-Borbón, L.G., González-Vega, R.I., Rodríguez-Figueroa, J.C., Rosas-Burgos, E.C., HuertaOcampo, J.A., Ruiz-Cruz, S., Wong-Corral, F.J., BorboaFlores, J., Rueda-Puente, E.O., Del-Toro-Sánchez, C.L. 2018. Liberación de compuestos fenólicos ligados en el garbanzo (Cicer arietinum L.) utilizando microbiota humana intestinal. Biotecnia. 20(3):146-154.

Prior, R.L., Wu, X., Schaich, K. 2005. Standardized Methods for the determination of antioxidant capacity and phenolics in foods and dietary supplements. Journal of Agricultural and Food Chemistry. 53: 4290- 4302.

Rocha-Guzmán, N.E., González-Laredo, R.F., Ibarra-Pérez, F.J., Nava-Berumen, C.A., Gallegos-Infante, J.A. 2007. Effect of pressure cooking on the antioxidant activity of extracts from three common bean (Phaseolus vulgaris L.) cultivars. Food Chemistry. 100: 31-35.

Roginsky, V., Lissi, E.A. 2005. Review of methods to determine chain-breaking antioxidant activity in food. Food Chemistry. 92(2): 235-254.

Rubio, C.P., Hernández-Ruiz, J., Martínez-Sbuela, S., Tvarijonaviciute, A., Ceron, J.J. 2016. Spectrophotometric assays for total antioxidant capacity (TAC) in dog serum: an update. BMC Veterinary Research. 12(1): 166.

Saura-Calixto, F., Serrano, J., Goni, I. 2006. Intake and bioaccessibility of total polyphenols in a whole diet. Science Direct 101 492-501.
Scalbert, A., y Williamson, G. 2000. Dietary Intake and Bioavailability of Poliphenols. Journal Nutrition. 130 (5): 346-356.

Scalbert, A. C., y Morand, C. 2005. Dietary polyphenols and the prevention of diseases. Critical Reviews in Food Science and Nutrition. 25(2): 297-306.

Soriano, S.R.A., Pavan, V. Pastore C.M. 2015. Effect of in vitro digestion on bioactive compounds and antioxidant activity of common bean seed coats. Food Research International. 76: 74-78.

Surangi, H., Thilakarathna, H.P., y Rupasinghe, V. 2013. Flavonoid Bioavailability and Attempts for Bioavailability Enhancement. Nutrients. 5: 3367-3387.

Tarko, T., DudaChodak, A., Sroka, P., Satora, P., Michalik, J. 2009. Tranformations of Phenolic Compounds in an in vitro Model Simulating the Human Alimentary Tract. Food Technology and Biotechnology. 47(4): 456-463.

Van-Campen, D.R., Glahn R.P. 1999. Micronutrient bioavailability techniques: accuracy, problems and limitations. Field Crops Research. 60: 93-113.

Venu, P., Holm, D.G., Jayanty, S.S. 2012. Effects of cooking methods on polyphenols, pigments and antioxidant activity in potato tubers. Food Science and Technology. 45: 161-171.

Xu, B.J., Chang, S.K.C. 2009. Total phenolic, phenolic acid, anthocyanin, flavan-3-ol, and flavonol profiles and antioxidant properties of pinto and black beans (Phaseolus vulgaris L.) as affected by thermal processing. J Agric Food Chem. 57: 4754-4764.

Yokozawa, T., Chen, C.P., Dong, E., Tanaka, T., Nonaka, G.I., Nishioka I. 1998. Study on the inhibitory effect of tannins and flavonoids against the 1,1-diphenyl-2-picrylhydrazyl radical. Biochemical Pharmacology. 56(2): 213-222.

Yuwei, L, Weihua, X, Zhenping, H., Xiaoxiao, J., Qian, W. 2014. The impact of processing on in vitro bioactive compounds bioavailability and antioxidant activities in faba bean (Vicia faba L) and azuki bean (Vigna angularis L.). International Food Research Journal. 23(3): 995-1001. 